\title{
Receive Descriptor Recycling for Small Packet High Speed Ethernet Traffic
}

\author{
Cedric Walravens \\ Department of Electrical Engineering - ESAT \\ Katholieke Universiteit Leuven, Belgium \\ Email: c.walravens@ieee.org
}

\author{
Benjamin Gaidioz \\ CERN \\ Geneva, Switzerland \\ Email: benjamin.gaidioz@cern.ch
}

\begin{abstract}
This paper presents the concept of Receive Descriptor Recycling to significantly reduce the performance drop associated with small packet Gigabit Ethernet traffic. Since limits and trade-offs are inherent when optimising for small packet traffic, all important aspects in this context are covered. Lowlevel measurements were performed at the CERN LHCb online Data Acquisition (DAQ) system, which is to a large extend made up of commodity equipment. Results gathered show the Ethernet Controller (Network Interface Card, NIC) driver currently is the major bottleneck, preventing the system from reaching maximal Gigabit Ethernet performance. Receive Descriptor Recycling is implemented under Linux for Intel's e1000 NIC driver, and is shown to successfully remedy the driver inefficiency.
\end{abstract}

\section{INTRODUCTION}

Broadband technologies enable considerable amounts of data to be transferred through a large network at comfortable speeds. Many modern DAQ systems, such as the LHCb Online DAQ system [1], can therefore depend on commodity hardware to reduce cost and simplify administration. Gigabit Ethernet is particularly attractive because this technology matured over the years and it has a very appealing performance to cost of ownership ratio.

Apart from full load link traffic, of which the effects have been studied in [2] [3], small packets have become more and more important in many real time applications. An example of a popular application, that causes this kind of network traffic, is voice-over-IP (VoIP). For each VoIP connection, 50 network packets are sent every second with a data payload size of 160 bytes or even less [4]. All Linux based firewalls, routers and web caches, where a large amount of small network packets is traveling through, can benefit from the results of this paper.

High reliability of small-sized transmissions is also crucial for the correctness of calibration runs for the $\mathrm{LHCb}$ [5] experiment, at the CERN LHC accelerator [6]. Here, small size packets are combined with extreme high packet rates and a near real time constraint. Some work on small packet traffic effects was conducted in [7], but no thorough low-level analysis has yet been performed. Also, Intel has released a document on small packet traffic performance [8], however, it presents merely recommendations which are not substantiated by solid measurements to proof any performance gain.

This paper studies the performance of an Ethernet device with small packets and remedies the associated performance drop by implementing Receive Descriptor Recycling (RDR) for Intel's e1000 NIC Linux driver. First, Section II will elaborate on the LHCb DAQ system, where the low-level measurements are performed. Section III describes the hardware setup and equipment used, followed by the throughput performance measurements and low-level analysis in Section IV and V, respectively. Both gather proof implying the Ethernet Controller's driver as the actual bottleneck. Finally, a solution to this bottleneck is provided by the RDR mechanism described in Section VI. Section VII concludes the paper with a discussion on further work.

\section{LHCB DAQ ONLINE SySTEM}

The LHCb Data Acquisition network [1] relies on Gigabit Ethernet over copper UTP to interconnect custom made electronics with the computing farm of commodity PCs. The farm of processors is partitioned into sub-farms, each one being interfaced to the readout network switch by a gateway (called an Sub-Farm Controller, SFC). A schematic overview of the system is shown in Fig. 1.

Performance of a SFC is critical for sizing whole the system. It can be measured by means of packet rate, packet loss, response time and throughput. Also, efficient CPU utilisation and resource requirements are important criteria. When optimising for small packet traffic, inevitable trade-offs are encountered, e.g. CPU and resource requirements increase in order to accommodate high rates of small packets, along with a rise in host bus utilisation. Note that transmit performance is not affected by small packet traffic to the same extent as receive performance. This asymmetry exists because the local host cannot usually overwhelm the Ethernet Controller with outgoing traffic.

\section{HARDWARE SETUP}

All benchmarks were performed on a SFC candidate system running two 32-bit Intel Xeon processors. The DAQ LAN is a Gigabit Ethernet network, connected by cat 5e copper UTP wires. The setup contained two servers and one network processor [9] as a very powerful traffic generator. All servers had hyper-threading [10] enabled.

Both Intel Pro/1000 MT dual and quad port Ethernet NICs were used. On the SRV06 host, version 6.0.54-k2-NAPI of the e1000 network driver was used, on the SFC04 host this was version 5.6.10.1-k2-NAPI. An overview of relevant system 


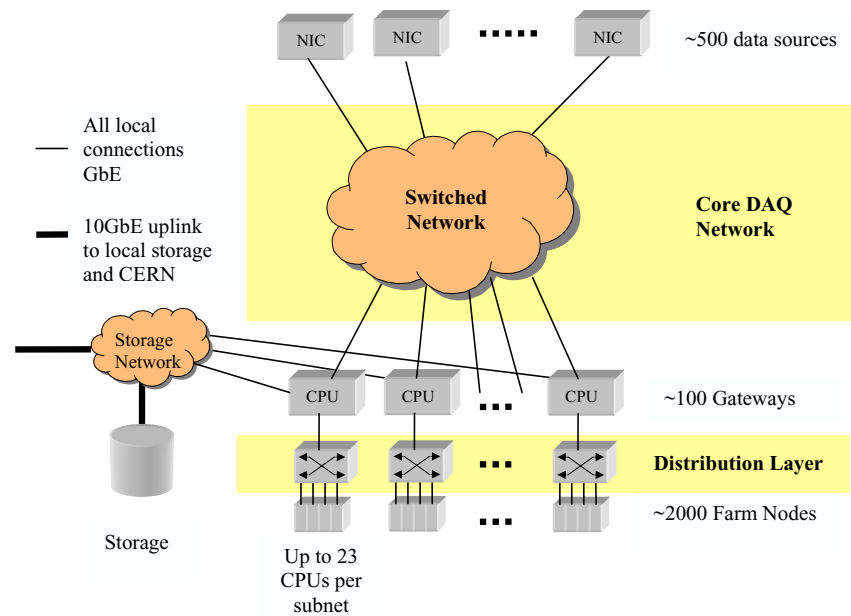

Fig. 1. Schematic view of the LHCb DAQ.

TABLE I

SPECIFICATIONS OF HARDWARE USED

\begin{tabular}{|c|c|c|c|c|c|}
\hline Host & $\begin{array}{l}\text { Chipset } \\
\text { PCI-X bus }\end{array}$ & \multicolumn{3}{|c|}{$\begin{array}{l}\text { CPU } \\
\text { Systembus }\end{array}$} & $\begin{array}{l}\text { Linux } \\
\text { Kernel }\end{array}$ \\
\hline 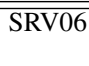 & $\begin{array}{l}\text { ServerWorks GC } \\
64 \text { bit } 133 \mathrm{MHz}\end{array}$ & \multicolumn{3}{|c|}{$\begin{array}{l}\text { Dual Xeon } 2.4 \mathrm{GHz} \\
400 \mathrm{MHz}\end{array}$} & $\begin{array}{l}\text { Scientific } 3.0 .4 \\
\text { 2.6.12-smp }\end{array}$ \\
\hline SFC06 & $\begin{array}{l}\text { Dell SC1425 } \\
64 \text { bit } 133 \mathrm{MHz}\end{array}$ & \multicolumn{3}{|c|}{$\begin{array}{l}\text { Dual Xeon } 2.8 \mathrm{GHz} \\
800 \mathrm{MHz}\end{array}$} & $\begin{array}{l}\text { Scientific } 3.0 .4 \\
2.6 .11 \text {-smp }\end{array}$ \\
\hline \multicolumn{2}{|l|}{ Type } & & Chipset & \multicolumn{2}{|c|}{ Host bus (max) } \\
\hline \multicolumn{2}{|c|}{$\begin{array}{l}\text { Intel Pro/1000MT } \\
\text { Intel Pro/1000MT } \\
\text { IBM PowerNP NP4GS3 }\end{array}$} & & $\begin{array}{l}82546 \mathrm{~EB} \\
82546 \mathrm{~EB} \\
\text { BCM5700 }\end{array}$ & \multicolumn{2}{|c|}{$\begin{array}{l}\text { PCI-X 64b 133MHz } \\
\text { PCI-X 64b 133MHz } \\
\text { PCI }\end{array}$} \\
\hline
\end{tabular}

specifications is presented in Table I, including the network processor used for frame generation.

The tests presented in this paper were performed using raw Ethernet frames using the IEEE 802.3 MAC format [11]; they consist of the 14 byte header followed by variable length user data and a 4 byte CRC-checksum at the end, resulting in a total data overhead of 18 bytes per frame.

\section{Receive Throughrut Performance}

To measure receive throughput, the network processor (NP) acted as a source, flooding the SFC with packets. Fig. 2(a) shows the bit rate measured when receiving on one port of the SFC from one port of the NP with a variable frame size. The bit rate measured at the output of the NP well matches the theory, apart from regular drops. According to the NP documentation, this behaviour is to be expected when pushing this device to the Gigabit Ethernet limit. The SFC receive rate drops accordingly of course. In the range of 0 to 200 , the bit rate measured at the input of the SFC is much lower (data is lost). In higher ranges, the sent bit rate is reached (no losses). Receiving on both ports of the dual card simultaneously, produces an identical plot.

Fig. 2(a) also shows the theoretical limit of the PCI bus. It is established that, in the case of operating one single port, this PCI bandwidth will not pose any limit on Gigabit Ethernet transfers, even taking into account the fact that this is

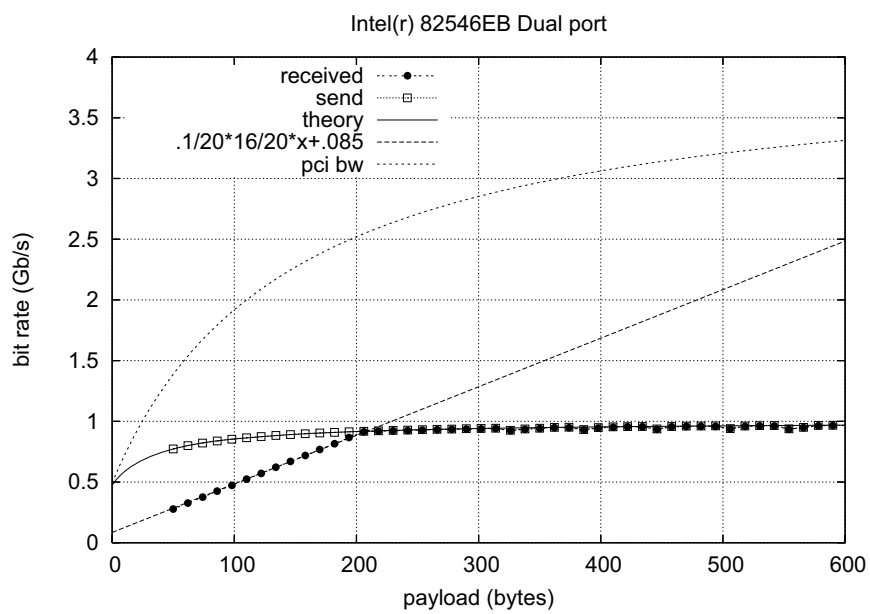

(a) Dual card, one port, plotted with theoretical Gigabit Ethernet limit, PCI bandwidth and slope.

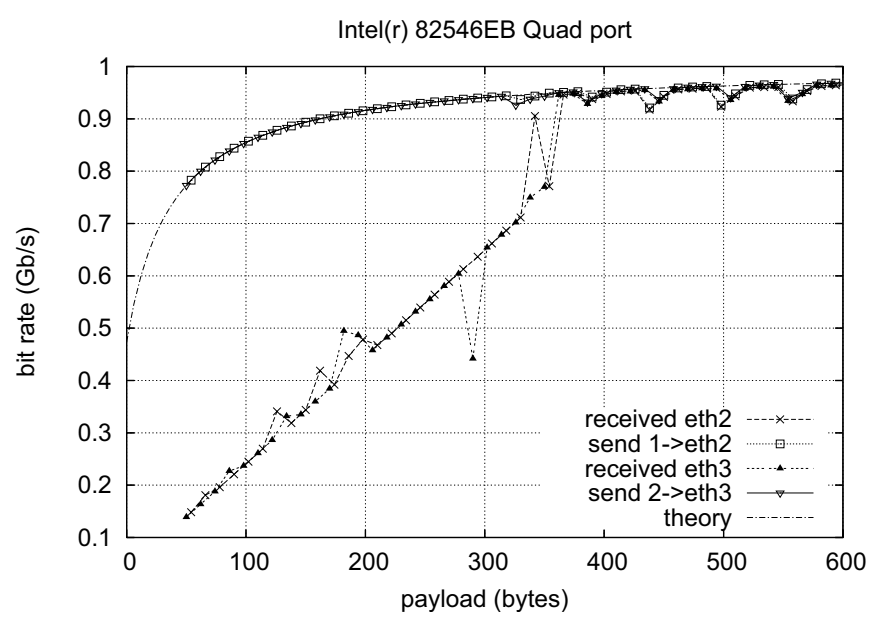

(b) Quad card, two ports simultaneously, plotted with theoretical Gigabit Ethernet limit.

Fig. 2. Receive throughput from NP (port $\mathrm{x}$ ) to SFC (eth $\mathrm{y}$ ) for Intel NIC.

a theoretical value which will never actually be reached due to numerous non-idealities. The same figure also shows a linear fit of the first part of the curve. The slope is 0.004 , which is in agreement with measurements in [7]. In the continuation of this paper, however, a different conclusion with regard to what contributes to this slope will be reached.

The same measurements were done on an Intel quad port NIC, the resulting plot is shown in Fig. 2(b). The slope of the linear part now amounts to 0.002 , a value insensitive to the inter-frame delay setting of the NP. This slope is clearly worse than the 0.004 slope of the dual card, indicating the quad card to be not as 'streamlined' as the single chip dual card. During this test, no PCI-X transfer bottleneck was observed. 


\section{Low LeVel Measurements}

The above-mentioned benchmark measures the real performance of the network, but generally does not allow for identification of bottlenecks present. Therefore, lower level tests as PCI-X traffic analysis and kernel profiling were subsequently carried out, emphasising the hardware and software, respectively.

\section{A. PCI/PCI-X Analysis}

Similar to network overhead issues, PCI/PCI-X bus protocols also impose penalties on small packet traffic. In addition to the actual data transfer, each bus transaction requires extra control cycles which introduce overhead (e.g., arbitration latency, address phases, attribute phases, wait states). Bus overhead costs become more pronounced with small packet traffic as bus control and wait cycles consume a larger fraction of each bus transaction.

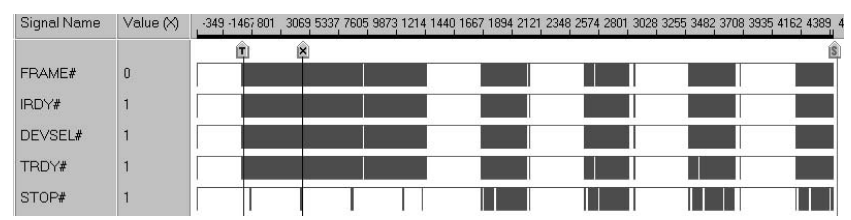

Fig. 3. Transition between smooth and stumble PCI traffic. (1 of 2 ports receiving from NP @ PCI 64bit 66MHz)

Fig. 3 shows the two regimes of transmission across the PCI bus when flooding the NIC with a NP. The dark areas indicate that the signal varies a lot, i.e. frames are transmitted across the bus. Notwithstanding that this plot was taken in PCI mode, exactly the same behaviour was observed when analysing the PCI-X traffic. This section summarises the flow of traffic on the bus, going from the smooth to the stumble regime.

During the smooth regime, all frames are nicely put on the PCI bus behind each other (inter-frame delay of 54 CLKs or $0.8 \mu \mathrm{s}$ ). The Intel Pro/1000 MT Ethernet Controller uses receive and transmit descriptors to keep the books [12]. Such a descriptor is basically a pointer to memory space, indicating a block of configurable size for the NIC to store a received frame or read in a frame to send. From here on, we will concentrate on reception of frames.

Receive Descriptors (RD) are made available to the NIC in groups of 16 consecutive descriptors. The tail address of this group is provided by a Receive Descriptor Tail (RDT). When a frame is received, the Ethernet Controller uses the next RD in the current RDT to find out where to store the frame. After the DMA transfer finishes, this RD is sent back to the driver to advertise the presence of a frame at that location (delay of 7 CLKs).

Fig. 4 visualises an interpreted version of Fig. 3. It shows left to right and top to bottom the advancing time axis (in clock ticks). The series of black dots are frames that are written to main memory. The following traffic pattern can be extracted for the smooth regime (0 to 14500 CLKs):
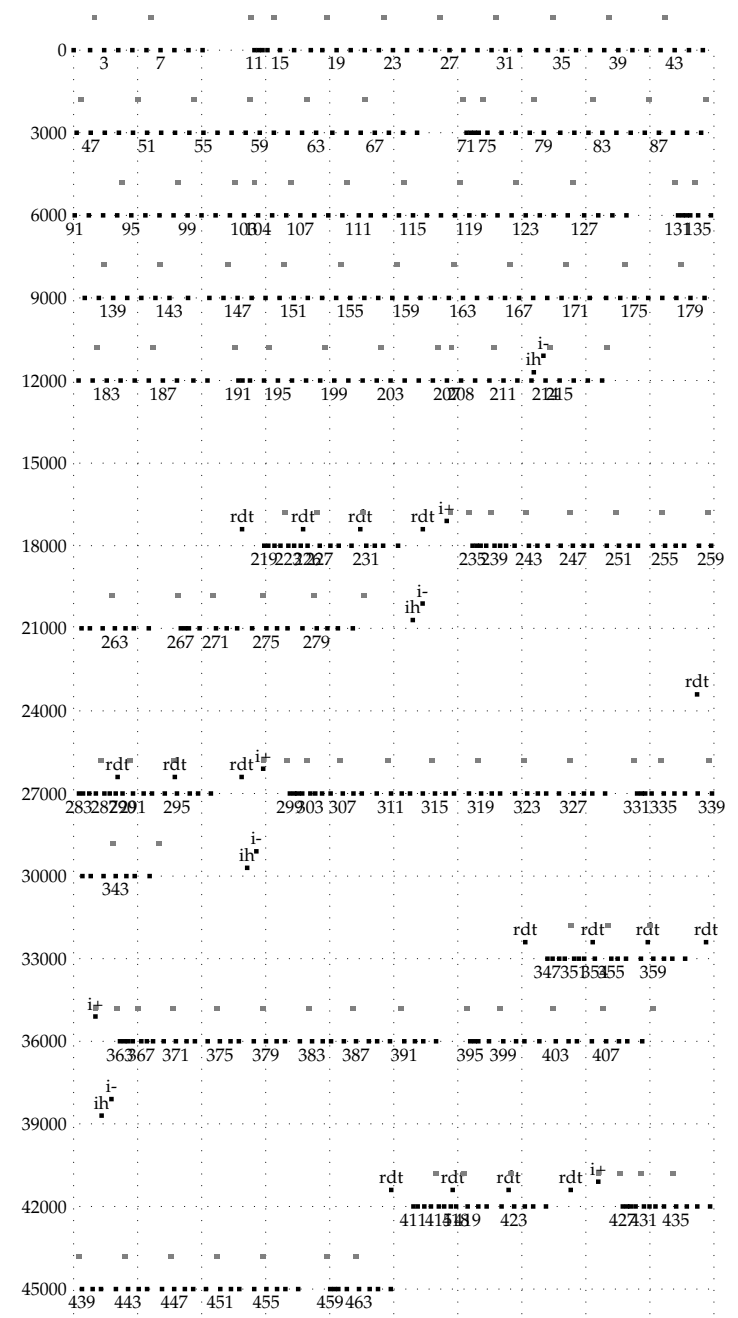

Fig. 4. Trace for the transmission from NP to SRV06 (from Fig. 3).

1) Four frames (black dots) are transferred to main memory through DMA bursts with an inter-frame delay of about 45 CLKs or $0.7 \mu \mathrm{s}$.

2) Following afterwards, i.e. 25 CLKs or $0.4 \mu \mathrm{s}$, the 4 corresponding RDs are written back to the driver (one grey spot).

3) Step 1 and 2 are repeated 16 times (containing a total of 64 frames), after which a new RDT is employed by the NIC, allowing to fetch 16 new RDs (small gaps in between series of 64 frames).

Under normal circumstances, the driver will provide the NIC with new RDTs (marked 'rdt' in Fig. 4), allowing it to fetch newly allocated RDs in time. This transfer of new RDs continues until all allocated descriptors for that batch have been provided, or, the card's frame receive buffer is nearly full and the card terminates the descriptor transfer by raising the STOP\# signal in order not to lose any frames. Since a NP is flooding the card during this test (with approximately 1 million packets per second), the latter will happen more and more, as 
seen on the STOP\# signal line (Fig. 3). After the descriptor transfer, the frame receive buffer is quickly emptied to regain the time lost during this transfer. All frames are now put on the PCI bus with a minimum delay of 7 CLKs in between.

Clearly, under persistent heavy traffic load, the card will become unable to fetch enough descriptors to keep up with the high frame rate, and at the same time sent every single frame to the system. The card will have to allow for a FIFO receive buffer overrun and silently drop frames until it has some more RDs to quickly empty the buffer and try start receiving again. The relief is, however, of short duration, as the card will soon have exhausted it (already) very limited pool of RDs, and it will have to wait again.

This is what happens in the second so-called stumble regime (14500 CLKs to end), where huge gaps of 4000 CLKs show the lack of RDs is preventing any further traffic until new RDTs are received. This results in many FIFO buffer overruns and a huge packet loss of up to $500 \mathrm{k}$ packets per second. Furthermore, the omnipresent STOP\# indicates that any transfer that takes longer than absolutely necessary is abruptly terminated by the NIC.

In the smooth part, the system can process 1 frame per microsecond (i.e. what the NP sends). When tumbling down into stumble regime, this number degrades to a value of merely 0.3 frames per microsecond or $0.28 \mathrm{~Gb} / \mathrm{s}$, cfr. earlier results, e.g. Fig. 2(a). This also confirms that the linear part for small packet sizes is caused by this stumble behaviour.

Where one might be tempted to think the PCI bus or NIC is the cause for this bottleneck, calculations on PCI bus utilisation proved otherwise. During the whole trace, peak data rates did not reach any higher than $250 \mathrm{MB} / \mathrm{s}$. Since the practical limit for a PCI bus is about $50 \%$ of the theoretical maximum, which is $533 \mathrm{MB} / \mathrm{s}$ in this case, this is acceptable and it was already a clear indication that nor the PCI bus, nor the card were responsible for this stumble behaviour. Note that this trace was taken for a single port receiving frames. It is clear that a PCI $64 \mathrm{bit} 66 \mathrm{MHz}$ will pose a bottleneck when more than 1 port and/or card is operating on the same bus.

Compared to PCI, it was observed that PCI-X traffic contained less errors and less accompanying WAIT states. Along with higher clock frequencies, the more intelligent use of WAIT states and so-called split transactions instead of delayed transactions are among the most important features of the PCI$\mathrm{X}$ protocol.

A look at the kernel's memory management information, provided by the /var/slabinfo file, made clear that memory access was responsible for the large gaps in stumble regime. These gaps can be explained by the DMA slab freeings. Normally, a CPU keeps a freed DMA slab in its bucket (one bucket per CPU). Occasionally, however, a large quantity of main memory is reallocated by the driver and then the main memory needs to be accessed, preventing others, e.g. DMA accesses by the NIC, from accessing the main memory. Beware, not the memory bank technology but the way the driver seems to handle memory access is causing the bottleneck. Simple on the back-of-the-envelope calculations show that the DDR-2 400MHz (PC2-3200) provides enough raw bandwidth.

It is clear that the Intel Pro/1000 MT Ethernet Controller card, when used in combination with a fast PCI-X host bus, will not become a bottleneck, even for quad port operation. Therefore, the next section takes a closer look at the software side of the network infrastructure, i.e. the Linux operating system and the e1000 Ethernet Controller driver.

\section{B. Kernel Profiling}

This last test employed the Oprofile package [13], a system-wide profiler for Linux systems, capable of profiling all running code at low overhead. It reports on the number of times a certain function (in user space, kernel, driver,...) has been called and what percentage of the total samples taken this represents.

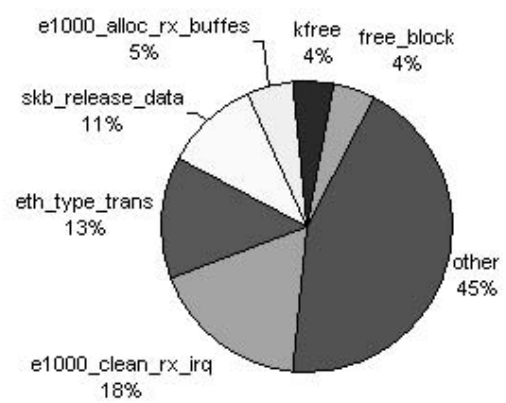

Fig. 5. Summary of relative occupation of the CPU during a 5 minute flood by the NP ( 1 of 2 ports @ PCI-X 64bit 133MHz).

Results of the OProfile test are summarised in Fig. 5. Analysing the exact content of the most frequently made calls, it is established that all are involve with freeing and reallocating RDs. This gave cause for the idea to tune the e1000 driver to more cleverly handle this RD processing, and implement some kind of Receive Descriptor recycling. This idea, along with its implementation in the e1000 source code, will be covered in the next section.

\section{RECEIVE DESCRIPTOR RECYCLING}

The e1000_main.c file of the e1000 driver source code [14] contains most of the driver's basic functionality. Analysing this source for packet reception handling on driver level, points out that the most frequently called functions of Fig. 5 are all related to one single very time consuming operation: the freeing of large heaps of memory. Especially when talking small payload sizes, it is clear that the situation only worsens due to more descriptors and thus more memory management overhead.

It is this overhead that prevents the driver from quickly sending new RDs to the Ethernet Controller, as they need to be freed and reallocated first. This is why the Receive Descriptor Recycling mechanism was implemented. The idea is to allocate a fixed number of permanent descriptors which are reused 
every time, effectively taking away the need for the costly reallocation overhead. The only processing that remains to be done is resetting some fields in the descriptors. The remainder of this section will outline the details of this implementation.

First it was needed to store the permanent buffers in the e1000_adapter struct (see e1000.h), so they became associated with each Ethernet Controller present in the system. For this, it was extended by two members: an array to store the pre-allocated socket buffers, and an array to point to the associated data fields. A fixed array size of 2048 was chosen, which was the RxDescriptors e1000 driver parameter used in all previous tests.

During the driver initialisation, the array is allocated in memory and the data pointers are set up. To prevent the driver from freeing the allocated array, the number of users for each socket buffer is artificially increased to 2 by calling skb_get ( ). As long as the number of users remains higher than 2, kfree_skb () will never free them, since it believes someone is still using the socket buffer and its data.

This is realised by altering the driver function call flow in e1000_alloc_rx_buffers (). Here the call to dev_alloc_skb(), which would return a fresh allocated socket buffer (skb), is replaced by returning one of our preallocated skbs in the array. Next, the skb reset is implemented by the newly added reset_skb () function.

Please refer to [15] for a detailed overview of exact code implementations.

To check for any performance increase for small packet sizes, the 'Receive Throughput' test (see Section IV) was repeated. For small frame sizes, the RDR-enabled driver was able to reduce packet loss by $40 \%$, see Fig. 6. The influence is the most clear for short delay packets. The performance of higher payloads remained unchanged with RDR.

\section{CONCLUSIONS AND FURTHER WORK}

Several benchmarks were performed on Gigabit Ethernet hardware and the Ethernet Controller driver. Among the aspects analysed in this work were throughput, packet loss and efficient resource utilisation. Emphasis was put on small packet size network traffic, to be used for calibration of the $\mathrm{LHCb}$ experiment. It was shown that the current bottleneck lies in the way the e1000 driver handles the Receive Descriptor memory management, which proofs to be fatal for small packet sizes. In order to remedy the situation, a Receive Descriptor Recycling mechanism was proposed and implemented in the official e1000 driver. Results have shown an improvement of small packet size performance by $40 \%$ in terms of increase in throughput and reduction of packet loss.

The knowledge gathered by the reported measurements, will be used to optimise the design of the DAQ infrastructure. The proposed Receive Descriptor Recycling will be tested in the real-life DAQ Prototype Farm environment. Further work also includes checking the possibility of turning off cache coherency by requesting non-cacheable buffer memory as to prevent numerous cache trashing.
NP->SRV06 - Intel dual card, two ports to NP - kernel 2.6.12

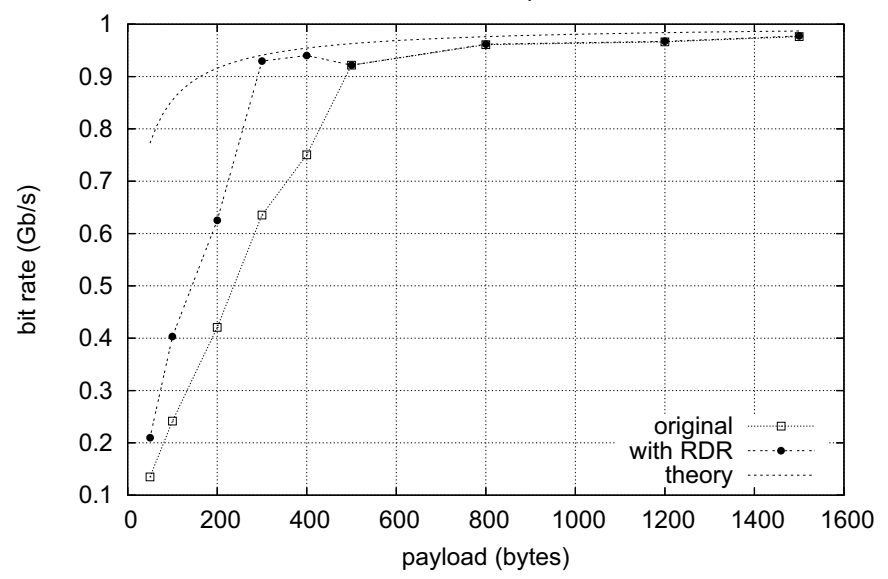

Fig. 6. Receive throughput from NP to SRV for two ports on a dual Intel NIC using official and patched driver.

\section{ACKNOWLEDGEMENTS}

This work has been funded by the Summer Student Internship Committee of CERN, Geneva, within the framework of the Summer Student Programme 2005.

\section{REFERENCES}

[1] A. Barczyk, J.-P. Dufey, B. Jost, and N. Neufeld, "The new LHCb trigger and DAQ strategy: A system architecture based on gigabit-ethernet," IEEE Trans. Nucl. Sci., vol. 51, pp. 456-460, June 2004.

[2] A. Barczyk, A. Carbone, J.-P. Dufey, D. Galli, B. Jost, U. Marconi, N. Neufeld, G. Peco, and V. Vagnoni, "Reliability of datagram transmission on gigabit ethernet at full link load," CERN, Geneva, CERN/LHCb 2004-030, 2004.

[3] R. Hughes-Jones, P. Clarke, and S. Dallison, "Performance of 1 and 10 gigabit ethernet cards with server quality motherboards," Future Generation Computer Systems, vol. 21, pp. 469-488, Apr. 2005.

[4] Voice Over IP - per call bandwidth consumption. [Online]. Available: http://cisco.com/warp/public/788/pkt-voice-general/bwidth_ consume.html

[5] LHCb, "LHCb technical proposal," CERN, Geneva, CERN/LHCC 98-4, 1998.

[6] O. S. Bruning, P. Collier, et al., Eds., LHC Design Report. Geneva, CH: CERN, 2004.

[7] A. Barczyk, D. Bortolotti, A. Carbone, J.-P. Dufey, D. Galli, B. Gaidioz, D. Gregori, B. Jost, U. Marconi, N. Neufeld, G. Peco, and V. Vagnoni, "High rate packets transmission on ethernet LAN using commodity hardware," IEEE Trans. Nucl. Sci., accepted for publication.

[8] "Small packet traffic performance optimization for $8255 \mathrm{x}$ and $8254 \mathrm{x}$ ethernet controllers," Application Note AP-453 rev. 1.0, Intel, Sept. 2003.

[9] J. R. Allen, Jr., et al., "IBM PowerNP network processor: Hardware, software, and applications," IBM J. Res. and Dev., vol. 47, Mar. 2003.

[10] "Hyper-threading technology on the Intel Xeon processor family for servers," White Paper, Intel, Oct. 2002.

[11] Ethernet LAN Medium Access Control (MAC) Specification, IEEE Std. 802.3, 1985.

[12] "PCI/PCI-X family of gigabit ethernet controllers software developer's manual," rev. 2.5, Intel, July 2005.

[13] OProfile development pages. [Online]. Available: http://oprofile. sourceforge.net

[14] e1000 source code at the Linux cross reference project. [Online]. Available: http://lxr.linux.no/source/drivers/net/e1000/

[15] C. Walravens and B. Gaidioz, "Low level gigabit ethernet analysis for the LHCb computing farm," CERN, Geneva, CERN/LHCb 2005-091. 